\title{
The Pros and Cons of Bringing Children up Bilingually
}

\author{
Rasool Mohammed Kheder \\ Department of English, College of Basic Education, University of Sulaimani, Sulaimani, Kurdistan Region - F.R. Iraq.
}

\begin{abstract}
The advisability of bringing children up bilingually is always questionable by the parents in general, but it is of vital importance for the parents of those children who are born within cross-national relationships. The current paper highlights the benefits and negative aspects of so doing from various points of view available in the related literature. It deals chiefly with assessing the parents' situations and suggesting appropriate approaches to them for decision making when they want to raise their children bilingually. Although qualitative method of research including the descriptive and argumentative methods are used for the majority parts of the study, the instances of the writer's bilingual experience are also mentioned as an outstanding proof in a case study. Whilst most of the common strategies for bringing children up bilingually are given in the current study, there are, of course, some other strategies for various circumstances. Other studies are recommended to focus mainly on strategies and reveal them for those parents who are eager to raise bilingual children.
\end{abstract}

Index Terms-Advisable Approaches, Advantages of Bilingualism, Bilingual Children Disadvantages of Bilingualism

\section{INTRODUCTION}

Bilingualism has a long history and it is widespread in many parts of the world. However, it has become an academic subject and has been studied from many different perspectives recently. It is still alive and an ongoing topic. There are many linguists, psychologists, teachers, researchers, and parents who are interested in bilingualism nowadays. This paper, mainly tries to focus on the parents who wonder about bringing their children up bilingually.

There are numerous different definitions available for the term bilingualism and bilingual in literature. Some of the most common ones will be mentioned in the current study briefly. Ducrot and Todorove (1972) state that, those individuals that acquired two languages as a first language and they master both languages perfectly are called bilinguals. Hoffmann (1991) stated several different considerations about bilinguals. For instance, we may say that technical translators are bilinguals, or we may say two-year-old children who speak in two different languages with their parents are bilinguals. We may also say that those young graduates that study a different language from their mother tongue for a long time are called bilinguals. Additionally, as cited by Bloomfield in Dewaele et al., (2003:177) bilingualism is defined as "native-like control of two or more languages". Therefore, taking all the above mentioned definitions into consideration, enables individuals to have various options of definitions to choose from and, as Skutnabb-Kangas (1981) states, choosing a definition of bilingualism depends on the use of individual's definition. In general, there is no accepted definition that is applicable to all linguists, psychologists, or researchers. Skutnabb-Kangas (ibid) categorized the bilingualism definitions into three major types: definitions that are based on competence, definitions that are based on function, and those definitions that are based on attitudes. Eventually, as Hoffmann (1991) argues the availability of many different definitions for bilingualism is not necessarily a problem, but on the contrary it gives the researcher the chance of choosing the most appropriate definition for her or his aims.

Now, it is worth looking into the relationship between the two languages and the mind. We, as human beings, always want to know what will happen in our minds when we are doing any activities. Especially most people are eager to know how the two or more languages work in the mind. As cited in Romaine (1995) and Johnson and Johnson (1999), Weinreich (1968) discussed three different kinds of bilingualism in terms of language encoding in the brain.

\section{1) Coordinate Bilingualism:}

In this type of bilingualism, the words of two languages will be kept separately in the brain of bilinguals. The languages in this type are independent and the individuals learn the languages in separate environments. As a researcher and a Kurdish-Persian bilingual, I may consider myself a particular instance here for clarification purposes. In that I acquired Kurdish as my mother tongue at home and the Persian language outside. Thereupon, the context I have acquired my languages are separate. Accordingly, a Kurdish concept Zanko /za:nko:/ and a Persian concept Daneshgah /da:ne Jga:h/ for 'university' word are kept separately in my mind and there is no connection between these two words from two different languages.

Journal of University of Human Development

Volume 5 No. 3(2019); DOI: 10.21928/juhd.v5n3y2019.pp73-78

Regular research paper: Published 16 July 2019

Corresponding author's e-mail: rasool.kheder@univsul.edu.iq

Copyright (C) 2019 Rasool Mohammed Kheder. This is an open access article distributed under the Creative Commons Attribution License

(CC BY-NC-ND 4.0) 


\section{2) Compound Bilingualism:}

In this type of bilingualism, the two languages will be learnt in the same environment. The languages are interdependent and they will become combined in the brain of this kind of bilingual. For instance, for a Kurdish-Persian bilingual that acquires both languages at home both single concepts of Zanko /za:nkP:/ and Daneshgah /da:ne $\mathrm{ga}: \mathrm{h} /$ refer to one common concept which is 'university' i.e. the bilinguals of this type have one concept for two different words from each language.

\section{3) Sub-ordinate Bilingualism:}

In the example of the Kurdish-Persian bilingual, if the Kurdish language is the dominant language, the word Daneshgah /da:nefga:h/ from the Persian language will be interpreted by the word Zanko /za:nkP:/ from Kurdish language. Ergo, according to this type of bilingualism, the individual bilingual has a primary set of meanings from her or his first language and another system of linguistics that is linked to them.

The age of acquisition is another crucial factor that parents should take into consideration when making decision to bring their children up bilingual. Harding and Riley (1986) suggest that we should distinguish some different ages that individuals become bilingual. They classified bilingualism in terms of age into infant bilingualism, child bilingualism, and later bilingualism. By infant bilingualism they mean those babies that acquire both languages from the beginning of their first steps of speaking. Child bilingualism refers to the successive acquisition of two languages particularly by those children that move to another country. Consequently, by late bilingualism they mean either adolescent bilingualism (people that become bilingual after puberty) or adulthood bilingualism (people that become bilingual after their teens). The importance of age classification for parents is to know the fact that in general, monolingual infants and children start speaking approximately sooner than bilingual ones. However, still the bilingual infants are able to start speaking at an acceptable age. Another fact is that the bilingual infants and children can approach the nativelike or even native accent of their second language, but bilingual adolescents and adults often have a non-native accent. Because of the importance of this subject, early and late bilingualism - from a different perspective - will be looked at in more details in later sections of the study.

\section{POSITIVE EFFECTS OF BILINGUALISM}

A substantial amount of studies that look at the positive effects of bilingualism are going on recently. Many researches show that children are encouraged to use two languages. As cited in Skutnabb-Kangas (1981), Cummins shows that bilingual children perform better than monolinguals in the areas of general intellectual development, divergent thinking, analysing various aspects of language, metalinguistic awareness, and sensitivity to non-verbal communication.

Vanpatten and Benati (2010) refer to three main types of advantages of being bilingual which have been studied and analyzed by scholars. These three types are: communicative, cultural and cognitive advantages. Also, Baker and Jones (1998) mention five fundamental communication advantages of bilinguals which are:
1. Relationships with parents: when parents have two different languages, their bilingual children are able to communicate in each parent's preferred language. This is a chance for them to develop a close relationship with their parents.

2. Extended family relationships: the bilinguals will be able to communicate with their relatives -their uncles, their aunts- when they live in different regions and they speak a different language from the local language.

3. Community relationships: unlike monolinguals, the bilinguals have the wider opportunities to build relationships with people of other nationalities.

4. Transnational communication: they have the potential of lowering the language barrier to create friendly relationships with other ethnic groups and nations.

5. Language sensitivity: bilinguals are more patient listeners than monolinguals when they meet people that they do not speak their language well.

Bilingualism has some cultural and economic advantages too. It is said that bilingualism means having two windows on the world. This means that bilinguals have the opportunity of experiencing two different cultures. According to Baker and Jones (1998), because bilinguals have knowledge of two different languages they will be able to penetrate and participate in the core of two different cultures. Thus, bilinguals have the potential of being bicultural, but this does not mean anyone who speaks two languages is in the core of two cultures. They also refer to the economic advantages of bilingualism and say those who know two languages probably have much job opportunities.

A good example among the studies regarding the cognitive advantages of bilingualism is the research study of Sheridan Scott who is mentioned in Hoffmann (1991). Scott wanted to find a connection between divergent thinking and bilingualism. Among two groups of children she realized that a group which wanted to become bilingual got higher scores in IQ levels than the other group that followed a conventional English language curriculum. She chose a group that were not bilingual, but were in a bilingual programme, because she wanted to know whether becoming bilingual has any effect on children's cognitive process. As mentioned above, Scott found that being bilingual has the advantage of having a higher level of IQ in comparison to monolingual children.

Achieving high levels of proficiency in both languages and positive effects on abilities to academic success are other important advantages of being bilingual. This is what Ellen Bialystok, cited in Lightbown and Spada (2006), and other developmental psychologists have found with convincing evidence.

\section{NEGATIVE EFFECTS OF BILINGUALISM}

Coping with two languages is very difficult for children. People who come up with this idea about bilingualism believe that children confuse two languages or they do not learn the second language well. To clarify this point this paper looks at 
some studies and presents whether these kinds of ideas are myths or facts of bilingualism.

Apple and Muysken (1987) state that, balance hypothesis is one of the negative views toward bilingualism. According to this hypothesis, the human capacity in terms of psychology, potential and neural, has some kinds of limitations for language learning. Hence, knowing one language limits learning the second language or other languages. However, Lightbown and Spada (2006) support a different view. In this sense, that there is no limitation in the human capacity to learn two or more languages. If we feel any limitation of bilingual languages, it does not mean that the individuals have the limitation in their capacity for learning more than one language. Language learning is more related to the environment and circumstances rather than human capacity.

The researcher's position in this argumentative issue, according to his experience of bilingualism, is the same with Lightbown and Spada (2006:26) who claim that, "There is no evidence that learning two languages substantially slows down their linguistic development". Sometimes I feel some limitation in my first language (Kurdish) regarding acceptable grammar structure. The reason behind this limitation is that, the language of instruction either in school or the university I studied at was not Kurdish. This means, I had more access with my second language (Persian) grammar rather than my first language. Therefore, it does not mean that I have certain restriction or limitation capacity for learning Kurdish grammar rules. But the environment in which I have studied had some impacts on my first language in terms of grammatical well-formedness.

\section{EARLY BILINGUALISM VS LATE BILINGUALISM}

Early and late bilingualisms are two terms that are used to distinguish between acquiring two languages in early childhood (early bilingualism) and learning them in adulthood (late bilingualism). Taylor and Taylor (1990) believe that, early bilinguals are those children that acquire their languages before the age of six, and late bilinguals are those individuals who learn their languages in adolescence or adulthood. Early bilinguals are more likely to acquire the languages before going to school - at home or outside - so they acquire languages unconsciously. That is why most of them are likely to obtain native-like proficiency in their second language. In some references the term child bilingualism has been used for early bilingualism. These two terms are used synonymously in this paper. Late bilinguals learn their second languages more formally and through conscious efforts.

Hoffmann (1991) refers to two opposite theories about early bilingualism which emerged in the 1970s. The first one is the unitary language system hypothesis. According to this theory bilingual children use one hybrid system at the beginning and then gradually this hybrid system will be separated. Therefore, at the first step they do not distinguish between the systems of two different languages. The second theory is the separate development hypothesis. This theory claims that bilingual children are able to differentiate between their two different languages very soon. Those who support this theory argue that there is no evidence to support the theory which says early bilinguals are unable to differentiate between language systems.

\section{Bilingual ChILDREN AND AdVISABLE APPROACHES}

As mentioned before, early bilingualism and child bilingualism terms have been used for the same concept in the current study. They both refer to either those children that have contact with two languages from their first months of their life, or those that acquire their second languages right away after their first language before about the age of six. What is important here is to know how children become bilingual, and identify the types of bilinguals.

This paper tries to shed some light on these questions first and then continues by looking at bilingual families in more detail.

Although there are lots of possible ways for children to become bilingual, this paper suffices with only five patterns that have been mentioned by Hoffmann (1991). He refers to five different patterns of how children become bilinguals and four different groups of bilinguals. They will be mentioned briefly as a guide to recognize bilingual families among those groups.

Pattern one. Immigration: This pattern refers to those children that leave their countries with their parents and stay in the host country either for a short or long period. They acquire their first language from their parents and from people of the host country where they acquire their second languages.

Pattern two. Migration: The children of this kind stay in the host country temporarily. Thus maintaining their first language is important for them. They may acquire their second language either from their parents or from the educational programs at school.

Pattern three. Common and close contact with different language groups: bilingualism is quite widespread in these kinds of communities. Children acquire their second language from their bilingual parents or outside the home from other members of community.

Pattern four. Schooling: Using second language as a medium of instruction, immersion courses, and private schemes for teaching minority languages, are examples of a schooling pattern for children to become bilingual. Since schooling or education play an essential role in bringing up bilingual children, bilingual education will be discussed in more details in later parts of this paper.

Pattern five. Bilingual families: This is another type of raising bilingual children. It is also, the pivot and mainstay of the current study which is considered as a separate section in later parts of the current study.

Now that it is clear which pattern we are looking for among the ways in which children become bilingual, it is important to know how many types of bilinguals are available and which one of them this paper will focus on.

Skutanabb-Kangas (1981) suggests dividing the world's bilinguals into four large groups, which are:

1. Elite bilinguals: those people who choose themselves freely to become bilingual such as students who want to study in other countries.

2. Children from linguistic majorities: this type refers to those children that learn other languages in the country they live. For example, those children from majority group that want to learn a language of minority groups at school in an immersion program. 
3. Children from bilingual families: this is a desirable type of bilingualism because children from this type are not under any pressure - except the pressure of becoming fluent in the official language - outside of their home. They are those children that grow up in a bilingual family and their parents speak different languages.

4. Children form linguistic minorities: a good example of this kind of bilingualism is, those Kurdish children who live in Iran and their parents belong to a linguistic minority. They are under strong external pressure to become bilingual, because Kurdish is not recognized as an official language in Iran.

Eventually after specifying, children from bilingual families among the four large groups of bilinguals, and schooling and bilingual families among mentioned patterns of bilinguals as choices and delimitations of this paper, it is time to discuss bilingual families and advisable approaches. Harding and Riley (1986) mention five types of bilingual families and Romaine (1995) added one more type to them. All the six types with a description of their characteristics and also the available studies for some particular languages will be referred to together within four major strategies in this paper. However, the term strategy is used instead of the type by the researcher and it is tried to answer a general question - what are the strategies parents use for bringing their children up bilingually? The question could be answered through discussing four different strategies below.

Strategy One:

This strategy is used when each parent speaks his or her native language to the child (from birth). This kind of strategy will be used in three different circumstances.

1. Each parent has a different native language from another but they both have some degree of competence from each other's languages. The language of the community is the dominant language of one of the parents.

2. The strategy also will be used when both of the parents use the same native language at home, but the dominant community language is different from that of the parents.

3. The last type of bilingual families that use this strategy is when each parent has his or her native language, and the dominant community language is not the same as either of the parents' languages.

Strategy Two:

The strategy is that the child will be exposed to the dominant community language in nursery school or outside home in general. For his or her first language input the parents, both speak with him or her in the non-dominant language. The type of bilingual families that use this strategy is more like the first type of bilingual family in strategy one but the strategy is different. Thus the strategy is used when each parent has a different native language and the dominant language of the community is the language of one of them.

\section{Strategy Three:}

The strategy is that the nonnative language always will be used by one of the parents. The parents who use this strategy have the same native language and the dominant community language is also the same with the language they use.

\section{Strategy Four:}

This strategy is added by Romaine (1995) to the above mentioned strategies by Harding and Riley (1986). The strategy is that both parents do code-switching and language mixing. This kind of strategy will be used when both parents and also maybe the community sectors are bilingual. As Romaine (1995) says this strategy leads to interference and mixing by children more than other strategies. That is why it was not mentioned by Harding and Riley (1986). They mentioned only the most effective and successful methods for raising bilingual children.

Although all the strategies mentioned above are the most common ones for bringing up bilingual children in the world, yet maybe none of them corresponds to our situations. Therefore, still there are some other strategies that parents can use for raising their children bilingually. They know better than anyone else, which strategy is the most appropriate one for their circumstances. Without any doubt there are many other strategies in the world for this issue, but because of researching difficulties in this field they are not featured in the literature so far. Also, there are many case studies and researches that demonstrate the above strategies, but because of limitations of this paper they will not be mentioned in detail. Instead of referring to all of them separately, the study will have a quick glance at some of them in general.

As cited in Romaine (1995), Ronjat and Leopold had done the earliest studies on their children about childhood bilingualism. Their studies and others are all based on extensive interviewing and observations. Baker (2006) says that there are some different approaches for raising children bilingually. He also refers to some terms such as shifting balance, and passive competence about bilingual children. One of the approaches introduced by Ronjat is the concept of one parent - one language. Ronjat's case study described a family in which the mother was speaking German, the father was speaking French, and the community language was French. This case study suggested that for bringing children up bilingually, the most effective way is that each parent speaks a different language to his/her child. The danger of this model is that, it may cause some limitations of the amount of discussion at home. Since parents have the most language influence in this model, any other external language effect such as family friends and carers may have the effect on the parents' plan. In the second approach parents speak in a minority language with their child who will acquire his or her second language in the community. The third approach is when both bilingual parents in a bilingual community speak both their languages with their child.

According to Baker (2006), shifting balance of the two languages by children is one of the concepts in some case studies. Leopold's case study was about his daughter Hildegard. Hildegard's father was speaking German and her mother was speaking English with her at home. Her languages were shifting when she went to Germany and came back to the United States. In Germany her German became stronger but when she came back to the United States her English became the dominant language. Shifting balance in bilingualism is also referred to among examples of Fantini (1985), Yukawa (1997), De Houwer (2003) and Quay (2001). As cited in Baker (2006:104), 
"De Houwer (2003) found that among some 2500 bilingual families, 1 in 5 children reared bilingually do not later use one of those languages". Quay (2001) agrees with De Houwer, but he uses the term passive competence in addition to his finding. Passive competence will be available for the weaker language by bilingualism and it can be used in other stages more actively. What is interesting about passive competence is that the bilinguals do not forget their languages completely when they do not use one of their languages. Even before starting to conduct this study the researcher - as a Kurdish-Persian bilingual - was believed that, he would mislays his Persian language in the near future, because he does not use it for a long period each year. But when he lives in a Persian-speaking community he can remember each single word in Persian easily. This is called productive competence by De Houwer (2005). He says that passive competence of the bilingual language will be changed very soon and quite rapidly to productive competence when the amount of input is increased and the bilingual felt the need to use that language (Baker, 2006).

\section{ASSESSing Situation AND DeCISION MAKING}

This part of the paper guides the parents on how to make their decisions about their children's languages. Parents are divided into two different groups. The first group of parents is ready for bringing their children up bilingually at birth. They are mostly aware of bilingualism and its features, and they have already made a plan for their children to speak in two different languages. The second group is parents that had missed the chance of raising their children bilingually before they become older than about six years. For the first group, following Harding and Riley (1968) the important factors that influence the parents' decisions whether to bring their children up bilingually or monolingual will be referred to.

Harding and Riley (1968) suggest that parents should ask themselves approximately nine important questions for assessing their situations in order to bring their children up bilingually or not. The questions are:

The parents should ask themselves about their language background. If they are bilinguals, certainly they have enough information about this issue and they treat with bilingualism as a natural phenomenon.

They should ask themselves that in which language(s) they speak together? They should be aware of different outcomes in different conditions. Is the language they use at home the same with the language of outside community or not? Do they speak in a language which is not the native language of either of them? For instance, the outcome of using the language of the outside community at home is that you need lots of time and numerous amount of effort for educating your child's languages.

Another important factor that determines their decision is the way of using their respective languages. The parents for example ask themselves, whether they will continue to speak in their mother tongues if they had different languages? Or they have some other plans for using their languages.

They should have a clear answer to the question like, is there anybody that look after their children when they are working? If yes, how he or she can affect the parents plan for their children's languages?
The parents should ask themselves about their attitudes towards their languages. Do they really mind leaving their mother tongue behind or they would prefer to speak with their children in their first languages?

Family relationships can play a remarkable role in making decision about bilingualism. Parents should ask themselves some questions about their contacts with their relatives. Do they have close contacts with the rest of their family? Will their children's languages be influenced by grandparents or cousins?

They should be aware of various statuses between languages. Are the languages they concerned in different status?

As Harding and Riley (1968:78) say from "the general climate and the availability of materials" viewpoints some pair languages are more difficult than others for maintaining. Parents should know about the availability of supporting means for maintaining the language.

The parents should ask themselves about changing their communication ways. Do they need to change the language that has been established as a habit for them? Are they aware about the impossibility of changing the established language to a new language? In this case, they should be knowledgeable enough to use some other patterns for establishing a bilingual family.

\section{EDUCATIONAL PROGRAMS FOR BILINGUALS}

As mentioned in the previous section, there are a group of parents that have missed the chance of bringing their children up bilingually before the age of six. Those parents, because of any reason, want their children become bilingual after they are six years or above. Bilingual education is the most common way for them. According to Huffmann (1991), there is no reason or study to support the idea that early bilingualism is better than late bilingualism. Therefore, when there is no chance for parents to raise their children bilingually from their birth, they still have the chance of using bilingual education. Another reason that magnifies the importance of bilingual education is that, most of the people are able to learn a second or even more languages at any time of their lives.

The term bilingual education has different meanings in different contexts. According to Skutnabb-Kangas (1981:121) the Anglo-American definition of bilingual education is that two languages should be used as mediums of instruction. So that, according to this definition most of the education immigrant children and also minority children which study in different countries will not be considered as bilinguals. Another definition of bilingual education which is more accurate is; the two languages in bilingual education should be used as means of instruction not as the languages themselves.

Romaine (1995) refers to some different types of bilingual educational policies which are:

1. Immersion: This type will be used for the purpose of enrich the majority of children in a bilingual program. Second language is a medium of teaching in this type of bilingual education.

2. Submersion: This type will be used for the purpose of assimilation. Sometimes without any additional second language teaching, minority children will put into majority language classes. Kurdish children in Iran, Turkey, and Syria are good examples of this type of bilingual 
education. They are placed in Persian, Turkish, and Arabic classes, and their use of their first language (Kurdish) is not permitted in the school campus.

3. Maintenance: This kind of program is sometimes called 'a language shelter'. First language is the medium of teaching in this program. Maintenance and development of the language and culture in interaction with the majority is main aim of this program.

Eventually, according to the above mentioned types of bilingual education, even sometimes without parents' wishes (They have no other chances for educating their children) their children become bilinguals, like Kurdish children example in submersion type. Additionally, there are some other cases in which parents choose the bilingual educational schools according to their preferences. Hence, using the bilingual schools is another chance for those parents that are eager to bring their children up bilingually.

\section{CONCLUSION}

The present paper revealed the following conclusions about the advantages, disadvantages, and advisable approaches for bringing up children bilingually:

Firstly, parents who missed the golden age of early bilingualism for raising their children bilingually, still have the chance of using the bilingual educational schools.

Secondly, parents should assess their contexts and then make their decision about the best strategies they can use. There are some other strategies that parents use according to their unique situation but are not exist in literature.

Finally, it is not a good idea to specify an approach and say this is the best approach for parents to use for raising their children bilingually. On the other hand, it is better to show the parents the ways of assessing their situations and let them choose the approaches they think are most appropriate for their contexts.

\section{REFERENCES}

Apple, R. and Muysken, P., (1987). Language Contact and Bilingualism. London: Edward Arnold.

Baker, C. and Jones, S.P., (1998). Encyclopedia of Bilingualism and Bilingual Education. Clevedon: Multilingual matters.

Baker, C., (2006). Foundations of Bilingual Education and Bilingualism.4th ed. Clevedon: Multilingual matters.

Dewaele, J.M. , Housen, A., and Wei, L., (2003). Bilingualism: Beyond Basic Principles. Clevedon: Multilingual Matters Ltd.

Harding, E and Riley, P., (1986). The Bilingual Family: A handbook for Parents. Cambridge: Cambridge university press.

Hoffmann, C., (1991). An Introduction to bilingualism, London: Longman.

Lightbown, P. M. and Spda, N., (2006). How Languages are Learned. Oxford: Oxford University Press.

Romaine, S., (1995). Bilingualism. 2nd ed. Oxford: Blackwell.

Skutnabb-Kangas, T., (1981). Bilingualism or Not: The Education of Minorities. Clevedon, Avon: Multilingual Matters.

Taylor, I., and Taylor, M. M., (1990). Psycholinguistics: Learning and Using Language. Prentice-Hall: Englewood Cliffs.

VanPatten, B., and Benati, A. G., (2010). Key Terms in Second Language Acquisition. York: Continuum International Publishing Group. 